\title{
Possible Evidence for Sparticles from Collider Data, and Some Implications
}

\author{
G.L. Kane ${ }^{1}$ \\ Randall Lab of Physics, University of Michigan, Ann Arbor, MI 48109-1120
}

\begin{abstract}
We examine the CDF ee $\gamma \gamma \mathbb{E}_{T}$ event as a candidate for sparticle production. Possible connections to other observables such as $R_{b}, \alpha_{s}, b \rightarrow s \gamma$, cold dark matter are briefly considered, and also implications for present and future data at FNAL and LEP, including the possibility that stops, gluinos and squarks are already being detected at FNAL. An analysis of the resulting Higgsino-like LSP as a cold dark matter candidate gives very encouraging results.
\end{abstract}

\section{INTRODUCTION}

The theoretical motivation for nature being supersymmetric on the weak scale is very strong. The possibility of unifying the SM forces, of relating them to gravity, and of explaining the Higgs mechanism are exciting. Perhaps the most important aspect of SUSY is connecting theory at the unification and Planck scales with experimentation at colliders. Close behind is that SUSY provides a non-baryonic cold dark matter candidate, which the SM does not.

There are several reasons why it is important to pursue all hints of direct sparticle production. (a) SUSY manifests itself in rather subtle ways, and often the confirmation of one signal can be a different one related by the theory. (b) If light sparticles exist there are major implications for utilization of FNAL and LEP, and for planning for future facilities. (c) Once there is data on sparticle masses and couplings we can test whether the LSP can indeed be a cold dark matter candidate. (d) Most important, perhaps, is that with data we can begin to construct the effective Lagrangian of the theory at the electroweak scale. Then we can relate it to the effective Lagrangian at the unification scale and begin to make detailed experimental contact with fundamental theories at the Planck scale.

Recently a candidate for sparticle production has been reported [1] by the CDF group. This has been interpreted in several ways [2-5] and later with

1) Based on work in collaboration with S. Ambrosanio, C. Kolda, G. Kribs, S. Martin, S. Mrenna, and J. Wells.

CP400, First Latin American Symposium on High Energy Physics/VII Mexican School of Particles and Fields, edited by D'Olivo/Klein-Kreisler/Mendez

(C) 1997 The American Institute of Physics 1-56396-686-7/97/\$10.00 
additional variations [6-8]. The main two paths are whether the LSP is the lightest neutralino [2], [9], or a nearly massless gravitino [2-7] or axino [8]. In the gravitino or axino case the LSP is not a candidate for cold dark matter, SUSY can have no effect on $R_{b}$ or $\alpha_{s}^{Z}$ or $B R(b \rightarrow s \gamma)$, and stops and gluinos are not being observed at FNAL. In the case where the lightest neutralino is the LSP (NLSP) the opposite holds for all of these observables, so it is very interesting to pursue this case in detail and that is what I will do for the remainder of this talk.

Recently the CDF group has suggested that the "positron" in the above event may be a $\tau$. That does not have much effect on the interpretation, because the main conclusions come from the presence of the photons and missing energy. The photons tell us that the second lightest neutralino is the one produced, and that it has a large branching ratio to the lightest neutralino and a photon. The missing energy constrains the masses, and is a marker for SUSY.

The SUSY Lagrangian depends on a number of parameters. Since all of them have the dimension of mass that should not be viewed as a weakness because at present we have no theory of the origin of mass parameters. Probably getting such a theory will depend on understanding how supersymmetry is broken. When there is no data on sparticle masses and couplings it is appropriate to make theoretical simplifying assumptions to reduce the number of parameters, but once there may be data it is important to measure the parameters and to see what patterns emerge. We will proceed by making no assumptions about soft-breaking parameters. In practice even though the full theory has over a hundred such parameters that is seldom a problem since any given observable depends on at most a few.

After describing the data and its implications we can examine to the properties of the "observed" LSP and whether it can be the cold dark matter of the universe. The answer is yes.

\section{NLSP INTERPRETATION OF THE $e e \gamma \gamma$ EVENT}

The CDF event [1] has a $36 \mathrm{GeV} e^{-}$, a $59 \mathrm{GeV} e^{+}$(or $\tau$ ), photons of 38 and $30 \mathrm{GeV}$, and $\mathbb{E}_{T}=53 \mathrm{GeV}$ (these are transverse energies). The first question of course is whether there can be a SM interpretation - the largest contribution thought of so far is that the event is $W(\rightarrow e \nu) W(\rightarrow e \nu) \gamma \gamma$. We [2] estimate the rate is a little over $10^{-4}$ for such an event in $100 \mathrm{pb}^{-1}$. Further, no other events have been reported with $\gamma \gamma$ accompanied by possible $W W$ decay products. On the other hand, $\sigma \times B R$ for selectron pair production is of order one event for $100 \mathrm{pb}^{-1}$ (more precisely, $\sigma \times B R$ is over $\frac{1}{2}$ for a significant part of parameter space) and the detection efficiency for such an event is $5-25 \%$

The SUSY interpretation is then $q \vec{q} \rightarrow \gamma, Z \rightarrow \tilde{e}^{+} \tilde{e}^{-}$, followed by each 


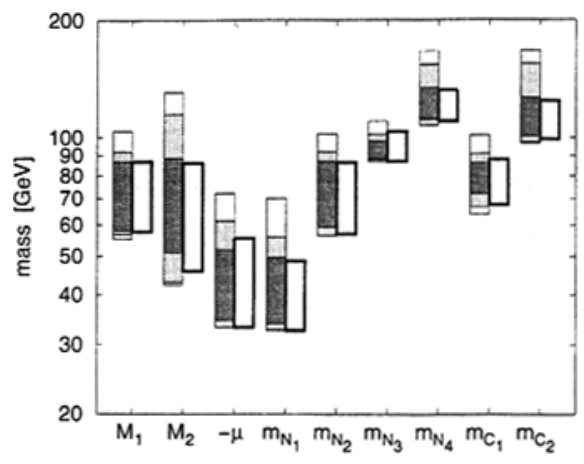

FIGURE 1. The allowed mass spectrum is shown for all models (shaded bands on the left) and for $\tilde{e}_{R}$ models only (thick solid outline on the right). The increasingly darker shades in the left-hand column correspond to increasing stricter cuts on $\mathcal{A}=5,7.5,10 \mathrm{fb}$. As for $\tan \beta$, the allowed range in all models is $1.0<\tan \beta<(2.8,2.6,1.8)$ for $\mathcal{A}=5,7.5,10 \mathrm{fb}$ respectively. The allowed range of $\tan \beta$ in $\tilde{e}_{R}$ models only is $1.0<\tan \beta<2.0 . \mathcal{A} \equiv \sigma B R^{2}$. $N_{i}$ are the four neutralino mass eigenstates and $C_{i}$ the two chargino mass eigenstates.

$\tilde{e}^{ \pm} \rightarrow e^{ \pm} N_{2}$, followed by $N_{2} \rightarrow \gamma N_{1}$. The second lightest neutralino, $N_{2}$, must be photino-like since it couples strongly to $\tilde{e} e$. Then the $\mathrm{LSP}=N_{1}$ must be higgsino-like [10-12] to have a large $B R\left(N_{2} \rightarrow N_{1} \gamma\right)$. Ref. 9 summarizes the resulting masses and couplings for charginos and neutralinos in detail, under various assumptions. Fig. 1 shows allowed ranges (keep in mind that values are correlated).

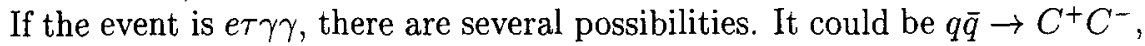
with $C^{+} \rightarrow W^{+}\left(\rightarrow \tau^{+} \nu\right) N_{2}\left(\rightarrow \gamma N_{1}\right), C^{-} \rightarrow W^{-}\left(\rightarrow e^{-} \nu\right) N_{2}\left(\rightarrow \gamma N_{1}\right)$, or $C^{+} \rightarrow \tilde{\tau}\left(\rightarrow \tau^{+} N_{2}\left(\rightarrow \gamma N_{1}\right)\right) \nu_{\tau}, C^{-} \rightarrow \tilde{e}\left(\rightarrow e N_{2}\left(\rightarrow \gamma N_{1}\right)\right) \nu_{e}$. The $W$ 's can be virtual.

\section{PREDICTIONS FOR FNAL, LEP}

If light superpartners indeed exist, as in Fig. 1, FNAL and LEP will produce thousands of them, and measure their properties very well. It will be important to ensure these facilities are fully utilized.

The first thing to check at FNAL is whether the produced selectron is $\tilde{e}_{L}$ or $\tilde{e}_{R}$. If $\tilde{e}_{L}$, then the charged current channel $u \bar{d} \rightarrow W^{+} \rightarrow \tilde{e}_{L} \tilde{\nu}$ has 5-10 times the rate of $\tilde{e}_{L}^{+} \tilde{e}_{L}^{-}$, so some events might be in the present sample. We expect $\tilde{e}_{L} \rightarrow e N_{2}\left(\rightarrow \gamma N_{1}\right)$. If $\tilde{\nu}$ is heavier than $C_{1}$, [9] $\tilde{\nu} \rightarrow e C_{1}$. If $m_{\bar{t}}<m_{C_{1}}$, then $C_{1} \rightarrow \tilde{t}\left(\rightarrow c N_{1}\right) b$ so $\tilde{\nu} \rightarrow e b c N_{1}$; if $m_{\tilde{t}}>m_{C_{1}}$ then $C_{1} \rightarrow W^{*}(\rightarrow j j) N_{1}$ so $\tilde{\nu} \rightarrow e j j N_{1}$, where $j=u, d, s, c$. Either way, dominantly $\tilde{e}_{L} \tilde{\nu} \rightarrow e e \gamma J J E_{T}$ where $J$ may be light or heavy quarks. If no such signal appears probably 
the produced selection was $\tilde{e}_{R}$. Also, $\sigma(\tilde{\nu} \tilde{\nu}) \cong \sigma\left(\tilde{e}_{L} \tilde{e}_{L}\right)$. Alternatively, if $\tilde{\nu}$ is lighter than $C_{1}, \tilde{\nu} \rightarrow N_{1} \nu$ and is invisible (or $\tilde{\nu} \rightarrow N_{2} \nu$ if $N_{1}$ has no zino component).

The most interesting channel at FNAL is $u \bar{d} \rightarrow W^{+} \rightarrow C_{i}^{+} N_{2}$. This gives a signature $\gamma J J \mathscr{E}_{T}$, for which there is no parton-level SM background. If $\tilde{t}<C_{i}$ one of $J$ is a $b$. If $\tilde{\nu}<C_{i}$ then $C_{i}^{ \pm} \rightarrow \tilde{\nu} \ell^{ \pm}$also. If $t \rightarrow \tilde{t} N_{2}$ (expected about $10 \%$ of the time) and if $\tilde{q}$ are produced at FNAL there are additional sources of such events (see below). They could be the best way to confirm SUSY in existing data. Fig. 2 shows the cross sections for charginos and neutralinos at FNAL.
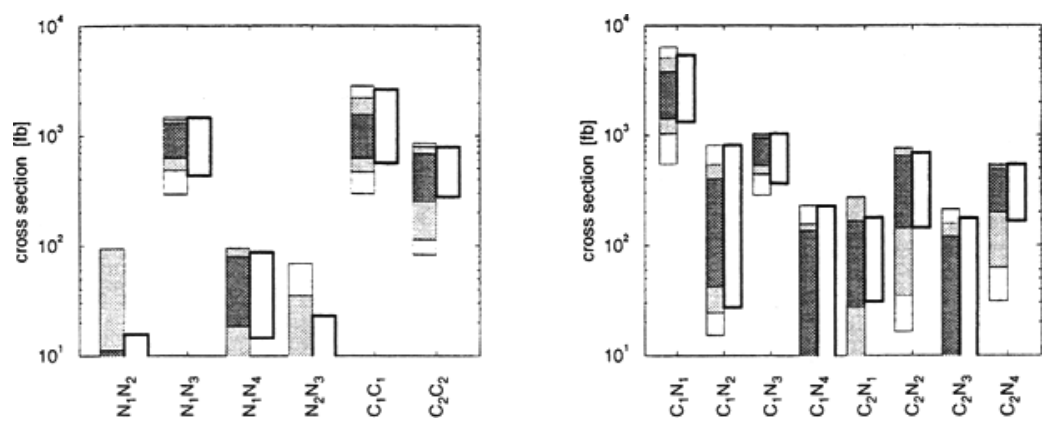

FIGURE 2. As in Fig. 1 , for Tevatron $\sqrt{s}=1.8 \mathrm{TeV}$ with all $\tilde{C}_{i}^{ \pm} \tilde{C}_{j}^{\mp}, \tilde{N}_{i} \tilde{N}_{j}, \tilde{C}_{i}^{ \pm} \tilde{N}_{j}$ processes shown that can have cross sections larger than about $50 \mathrm{fb}$.

Several processes could occur at LEP161, and many must occur at LEP190 [9]. The most likely one is $e^{+} e^{-} \rightarrow N_{1} N_{3}\left(\rightarrow Z^{*}(\rightarrow q \bar{q}) N_{1}\right)$, giving $q \bar{q} \not \mathbf{E}$ events. The $m(q \bar{q})$ is at most $m_{N_{3}}-m_{N_{1}} \lesssim 65 \mathrm{GeV}$. However, if $N_{3}$ is heavier than $\tilde{\nu}, N_{3} \rightarrow \tilde{\nu} \nu$ dominates. Then if $C_{1}$ is heavier than $\tilde{\nu}, \tilde{\nu}$ may decay invisibly to $N_{1} \nu$, or to $N_{2} \nu$ followed by $N_{2} \rightarrow N_{1} \gamma$. If the $C_{1}$ mass is in the allowed region $e^{+} e^{-} \rightarrow C_{1}^{+} C_{1}^{-}$will occur, often giving $J J J J \not$ events $\left(b \bar{b} c \bar{c}\right.$ if $\tilde{t}<C_{1}$, otherwise $j j j j$ with $j=u, d, s, c)$. However, if $C_{1}$ is heavier than $\tilde{\nu}$ other channels are open.

The process $e^{+} e^{-} \rightarrow N_{2} N_{2}$ gives $\gamma \gamma \notin$ with missing mass $\gtrsim m_{Z}$. Fig. 3 shows the LEP cross sections. This has a very good signature. It is very interesting, because the neutralino-LSP world gives excess events in this channel, all with invariant missing mass above the $Z$, while the gravitino-LSP world gives events with invariant missing mass of any value. There should be no background below the $Z$, so any confirmed events there both imply new physics and the very light LSP world. There is some background above the $Z$, but a substantial number of events there would imply new physics and the neutralino-LSP world. 

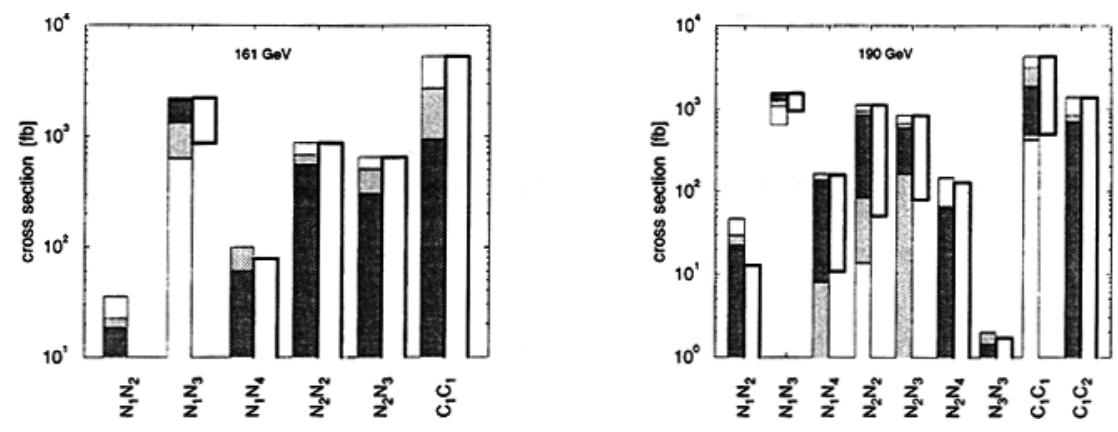

FIGURE 3. The range of the non-negligible cross sections at LEP161 and LEP190.

\section{GLUINOS, SQUARKS AT FNAL?}

If charginos and neutralinos and selectrons are light then gluinos and squarks should not be too heavy. If stops are light, then $B R\left(t \rightarrow \tilde{t} N_{i}\right) \lesssim 1 / 2$ [13], in which case extra tops must be produced because $\sigma \times B R(t \rightarrow W b)$ is near or above its SM value with $B R(t \rightarrow W b)=1$. With these motivations, we [14] have suggested that one assume $m_{\tilde{g}} \geq m_{t}+m_{\tilde{t}}$ and $m_{\tilde{q}} \geq m_{\tilde{g}}$. Then there are several $\mathrm{pb}$ of top production via channels $\tilde{q} \tilde{g}, \tilde{g} \tilde{g}, \tilde{q} \overline{\tilde{q}}$ with $\tilde{q} \rightarrow q \tilde{g}$, and $\tilde{g} \rightarrow t \tilde{t}$ since $t \tilde{t}$ is the gluino's only two-body $B R$. Analysis shows that this scenario is at least as consistent as the SM with published data, and perhaps more so in that the distribution for $P_{T}(t \bar{t})$ should peak at small $P_{T}$ for the SM but at larger $P_{T}$ for the SUSY case since the system is recoiling against extra jets in the SUSY case. To be quantitative, the fraction of events expected for $P_{T}(t \bar{t})>15 \mathrm{GeV}$ is $35 \%$ for the SM vs. $63 \%$ for SUSY; the reported CDF data has $71 \%$ beyond $P_{T}(t \bar{t})=15 \mathrm{GeV}$. The SUSY case suggests that if $m_{t}$ or $\sigma_{t \bar{t}}$ are measured in different channels one will obtain different values, which is also consistent with reported data. This analysis also shows that the present data is consistent with $B R\left(t \rightarrow \tilde{t} N_{i}\right)=1 / 2$. That this analysis does not lead to contradictions with data adds additional support to the whole picture.

$$
R_{b}, \alpha_{s}, B R(b \rightarrow s \gamma)
$$

At present $R_{b}$ and $B R(b \rightarrow s \gamma)$ differ from their SM predictions by $1.5-2 \sigma$, and (in my opinion) $\alpha_{s}$ measured by the $Z$ width differs by about $1.5-2 \sigma$ from its value measured other ways. If these effects are real they can be explained by $C_{i}-\tilde{t}$ loops [16]. What is particularly relevant here, and exciting, is that they can be explained by precisely the SUSY parameters deduced from the $e e \gamma \gamma$ event (+ a light, mainly right handed, stop). Although $\tan \beta, \mu$, and $M_{2}$ a priori could be anything, they come out the same from the analysis of these loops and from ee $\gamma \gamma\left(\tan \beta \leq 1.5, \mu \sim-m_{Z} / 2, M_{2} \sim 60-80 \mathrm{GeV}\right)$. While 
we cannot fully interpret this until these effects are better determined, this agreement is very encouraging.

\section{COLD DARK MATTER}

The LSP $=N_{1}$ apparently escapes the CDF detector in the ee $\gamma \gamma$ event, suggesting it is stable (though only proving it lives longer than $\sim 10^{-8} \mathrm{sec}$ ). If so it is a candidate for cold dark matter (CDM). The properties of $N_{1}$ are deduced from the analysis [9] so the calculation of the relic density [17] is a "no-parameter" one. The analysis shows that the s-channel annihilation through the $Z$ dominates, so the needed parameters are $\tan \beta, m_{N_{1}}$ and the higgsino fraction for $N_{1}$, which is large. The results are very encouraging, giving $0.1 \leq \Omega h^{2} \leq 1$, as shown in Fig. 4 .

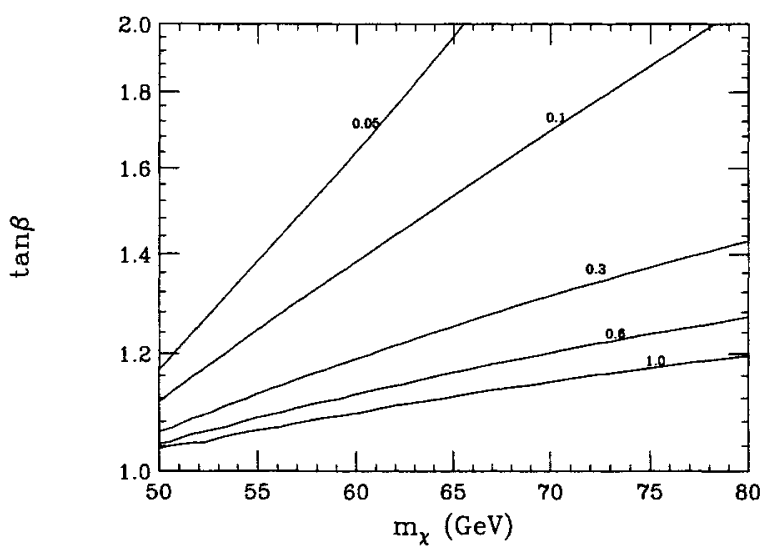

FIGURE 4. Contours of constant $\Omega h^{2}$ for the Higgsino-like LSP described in the text (solid).

The central value is $\Omega h^{2} \simeq 1 / 4$. Thus the CDM of the universe may have been observed at FNAL - the particle that makes up the CDM is approximately the superpartner of the Higgs boson. Such calculations can give $\Omega h^{2}$ very large compared to 1 or very small; that this gives about the right relic density for a flat universe is further encouragement that the entire picture may be correct.

At FNAL, of course, it can be demonstrated that $N_{1}$ escapes the detector but not that it is stable. We have checked [17] that the prospects for detecting $N_{1}$ in "direct" experiments are not bad. For example, in ${ }^{73} \mathrm{Ge}$ events would be seen in the worst case at a few hundred kg-days, and for some values of $m_{N_{1}}$ and $\tan \beta$ in the preferred region the event rate could be 10 times larger. Flourine and other nuclei give larger rates. While such rates are not large, they are probably eventually detectable in direct experiments, and the direct detection of cold dark matter is so important that is is worth a lot of effort. 
Freese and Kamionkowski [18] have looked at detection via $N_{1}$ annihilation to energetic neutrinos in the Sun, and conclude the rate for underground detectors could be within an order of magnitude of today's limits. See also the recent paper of ref. [19] for study of labroatory detections of the LSP; however, beware of increased rates from rescaling cases with small $\Omega h^{2}$.

\section{SUMMARY}

The ee $\gamma \gamma$ (or $e \tau \gamma \gamma$ ) event may be the first direct observation of superpartners. It is encouraging that the higgsino-like LSP deduced from the analysis of the event gives a relic density $\Omega h^{2} \sim 1 / 4$, that one can explain the 1.5-2 $\sigma$ effects in $R_{b}, \alpha_{s}$, and $B R(b \rightarrow s \gamma\}$, and that there may be additional evidence for $\bar{t}, \tilde{g}$, and $\tilde{q}$ production at FNAL. The consistency of this picture need not have occurred. We have given a number of predictions for existing and future FNAL and LEP data to test the correctness of this picture.

\section{ACKNOWLEDGMENTS}

This research was supported in part by DoE. I am very grateful to my collaborators Sandro Ambrosanio, Graham Kribs, Steve Martin, Steve Mrenna and Jim Wells and to A. Blondel, A. Buras, M. Carena, S. Pokorski and C. Wagner for helpful conversations. I wold like to thank L. Diaz Cruz, M. Perez, A. Garcia, and R. Huerta very much for their warm hospitality.

\section{REFERENCES}

1. S. Park, "Search for New Phenomena in CDF", $10^{\text {th }}$ Topical Workshop on Proton-Anti-proton Collider Physics, edited by Rajendran Raja and John Yoh, AIP Conf. Proc. No. 357 (AIP, New York, 1996); L. Nodulman, "New Particle Searches at CDF", Proceedings of the International Europhysics Conference on High Energy Physics, edited by J. Lemonne et al. Brussels, 1995.

2. S. Ambrosanio, G. L. Kane, G. D. Kribs, S. P. Martin, and S. Mrenna, Phys. Rev. Lett. 76 (1996) 3498.

3. S. Dimopoulos, M. Dine, S. Raby, and S. Thomas, Phys. Rev. Lett. 76 (1996) 3494 .

4. S. Dimopoulos, S. Thomas, and J. D. Wells, Phys. Rev. D54 (1996) 3283.

5. S. Ambrosanio, G. L. Kane, G. D. Kribs, S. P. Martin, and S. Mrenna, Phys. Rev. D 54 (1996) 5395.

6. K. Babu, C. Kolda, and F. Wilczek, Phys. Rev. Lett. 77 (1996) 3070.

7. J. L. Lopez and D. V. Nanopoulos, Mod. Phys. Lett. A10 (1996) 2473.

8. J. Hisano, K. Tobe, and T. Yanagida, Phys. Rev. D55 (1997) 411.

9. S. Ambrosanio, G.L. Kane, G. Kribs, S. Martin, S. Mrenna, hep-ph/9607414. 
10. H. Komatsu and J. Kubo, Phys. Lett. 157B (1985) 90; Nucl. Phys. B263 (1986) $265 ;$ H. E. Haber, G. L. Kane, and M. Quirós, Phys. Lett. 160B (1985) 297; Nucl. Phys. B273 (1986) 333.

11. H. E. Haber and D. Wyler, Nucl. Phys. B323 (1989) 267.

12. S. Ambrosanio and B. Mele, Phys. Rev. D52 (1995) 3900; Phys. Rev. D53 (1996) 2541

S. Ambrosanio and B. Mele, ROME1-1148/96.

13. J.D. Wells, C. Kolda, G. L. Kane, Phys. Lett. B 338 (1994) 219.

14. G. L. Kane and S. Mrenna, Phys. Rev. Lett. 77 (1996) 3502.

15. See the Rapporteur talks of A. Blondel and A. Buras at the XXVIII International Conference on HEP, Warsaw, July 1996.

16. A. Djouadi et al. Nucl. Phys. B349 (1991) 48; M. Boulware, D. Finnell, Phys. Rev. D44 (1991) 2054; J. D. Wells, C. Kolda, G. L. Kane, Phys. Lett. B 338 (1994) 219; D. Garcia, J. Sola, Phys. Lett. B 354 (1995) 335; G. L. Kane, R. G. Stuart, J. D. Wells, Phys. Lett. B 354 (1995) 350; A. Dabelstein, W. Hollik, W. Mösle, hep-ph $/ 9506251$; M. Carena, H. E. Haber, and C. E. M. Wagner, hep-ph/9512446. P. H. Chankowski, S. Pokorski, Phys. Lett. B 366 (1996) 188; J. Ellis, J. Lopez, D. Nanopoulos, Phys. Lett. B 372 (1996) 95; J. D. Wells, G. L. Kane, Phys. Rev. Lett. 76 (1996) 869; E. H. Simmons, Y. Su, hepph/9602267; P. H. Chankowski, S. Pokorski, hep-ph/9603310.

17. G. L. Kane and J. D. Wells, Phys. Rev. Lett. 76 (1996) 4458.

18. K. Freese and M. Kamionkowski, hep-ph/9609370; K. Freese, private communication.

19. A. Bottino, N. Forneugo, G. MIgrida, M. Olechowski, and S. Scopel, astro$\mathrm{ph} / 9611030$. 